\title{
Intracoronary adiponectin at reperfusion reduces infarct size in a porcine myocardial infarction model
}

\author{
MARCIN DĘBIŃSKI ${ }^{1}$, PIOTR P. BUSZMAN ${ }^{1}$, KRZYSZTOF MILEWSKI $^{1,3}$, WOJCIECH WOJAKOWSKI ${ }^{1,2}$, \\ WANDA JACKIEWICZ ${ }^{1}$, JACEK PAJĄK ${ }^{2}$, DARIUSZ SZURLEJ ${ }^{2}$, JOANNA FRYC-STANEK ${ }^{2}$, \\ SZYMON WIERNEK $^{1,4}$, MICHAL JELONEK $^{1}$, MICHAEL E. SPURLOCK $^{5}$, JACK MARTIN $^{6}$, \\ ANDRZEJ BOCHENEK ${ }^{1,2}$ and PAWEL E. BUSZMAN ${ }^{1}$ \\ ${ }^{1}$ American Heart of Poland; ${ }^{2}$ Medical University of Silesia, Katowice, Poland; \\ ${ }^{3}$ Skirball Center For Cardiovascular Research, Cardiovascular Research Foundation, Orangeburg, NY; \\ ${ }^{4}$ San Antonio Endovascular and Heart Institute, San Antonio, TX; ${ }^{5}$ Iowa State University Departments of Animal Science, \\ Food Science and Human Nutrition, Nutrition and Wellness Research Center, Ames, IA; ${ }^{6}$ Sharpe-Strumia \\ Research Foundation of the Bryn Mawr Hospital, Jefferson Health System, Philadelphia, PA, USA
}

Received December 14, 2010; Accepted February 1, 2011

DOI: $10.3892 /$ ijmm.2011.643

\begin{abstract}
Reperfusion injury (RI) remains an important limitation of myocardial revascularization. The aim of the present study was to evaluate the influence of the intracoronary injection of adiponectin on RI and cardiomyocyte death in a porcine myocardial infarction model. Acute infarction in 14 Polish domestic pigs was induced by inflation of an over the wire balloon (OTW) catheter in the medial left anterior descending artery for $60 \mathrm{~min}$. The study group consisted of 7 pigs in which intracoronary adiponectin $(50 \mu \mathrm{g})$ was infused through the OTW catheter immediately before reperfusion. The control group $(n=7)$ was administered placebo. Animals were sacrificed after two days of follow-up. The infarct area (IA) was stained with tetrazoline and the area at risk (AAR) with intracoronary administration of Evans Blue dye before euthanasia. Hearts in each group had similar AARs $(46.2 \pm 9.9 \%$ vs. $48.4 \pm 6.2 \%$ of the whole myocardium, $\mathrm{p}=\mathrm{ns})$. The IA/AAR\% and IA were smaller in the study group when compared to the control $(24.7 \pm 4.0 \%$ vs. $45.3 \pm 22.5 \%, \mathrm{p}=0.005$; and $11.7 \pm 4.9 \%$ vs. $20.5 \pm 5.6 \%, p=0.01$, respectively). These outcomes corresponded well with the peak troponin levels after $12 \mathrm{~h}(109.9 \pm 60.9 \mathrm{ng} / \mathrm{ml}$ vs. $185.5 \pm 39.4 \mathrm{ng} / \mathrm{ml}, \mathrm{p}=0.017)$. After two days there was a significantly higher LVEF in
\end{abstract}

Correspondence to: Dr Marcin Dębiński, American Heart of Poland, Katowice, Poland

E-mail: dabbad@wp.pl

Abbreviations: STEMI, ST segment elevation myocardial infarction; ACS, acute coronary syndrome; RI, reperfusion injury; AAR, area at risk; IA, infarct area; pPCI, primary percutaneous coronary intervention; LVEF, left ventricle ejection fraction; OTW, over the wire catheter

Key words: myocardial infarction, ischemia, reperfusion injury, porcine, adiponectin the study group $(51.4 \pm 8.5 \%$ vs. $33.9 \pm 8.6 \%, \mathrm{p}=0.002)$. There was also a trend toward lower apoptosis enhancement in the viable myocardium in the study group $(3.11 \pm 2.3$ vs. $8.92 \pm 6.3$; $\mathrm{p}=0.07)$. The administration of adiponectin into the infarctrelated artery is safe and feasible. The treatment significantly reduced the infarct size.

\section{Introduction}

Current strategies of reperfusion in ST elevation myocardial infarction (STEMI) with primary percutaneous coronary intervention (pPCI) are highly efficient with a high periprocedural success rate and with the hospital mortality ranging from $3-7 \%$ (1-3). However, in long-term observations, almost $30 \%$ of these patients develop heart failure $(4,5)$ associated with substantial long-term mortality (6). This is mostly due to the formation of post-infarction scars and to left ventricular remodelling. Timely percutaneous coronary intervention (PCI) can significantly reduce the infarct size by $40 \%$, while $30-40 \%$ of the final infarct area can be attributed to lethal reperfusion injury (RI) (7). This kind of injury can significantly reduce the benefit of reperfusion in STEMI and since it is potentially preventable, new approaches are being currently tested in animal models and in clinical trials (8).

Reperfusion can be described as a double edge sword (9) because it leads to the necrosis of cardiac myocytes that were viable before the restoration of the blood flow. RI may result in myocyte cell death (lethal $\mathrm{RI}$ ), transient myocardial dysfunction (myocardial stunning, arrhythmias) or to a no-reflow phenomenon. As shown in experimental studies, RI is a chain reaction involving the generation of reactive oxygen compounds and increased oxidative stress, disruption of the sarcoplasmic reticulum and intracellular and mitochondrial calcium overload, rapid changes of $\mathrm{pH}$ and release of lactate and finally activation of an inflammatory reaction. It is likely that the key event in the pathophysiology of RI is the opening of the mitochondrial permeability transition pore (PTP) leading 
to a reduction of ATP levels and to cardiomyocyte necrosis, as a result of the above-mentioned metabolic changes (10).

Many pharmacological agents and various techniques, such as preconditioning and intracoronary drug delivery, have been studied in order to reduce the burden of RI. The results of the clinical trials are inconsistent and only those pharmacological agents targeting several mediators in the RI pathway have shown to be successful in reducing infarct size $(11,12)$. Furthermore, none of them have been introduced into the clinical practice.

Currently, the fatty tissue-derived hormone, adiponectin, has emerged as a promising novel agent which may alleviate the problem of RI. It has been demonstrated that it increases the release of endogenous nitric oxide (13), inhibits the synthesis of oxygen free radicals $(14,15)$ and adhesion molecules (16). Furthermore, adiponectin can inhibit the apoptosis pathway, reduce caspase 3 activity (17), and possibly influence clot formation. Finally, mouse and rat experiments have shown its efficacy in myocardial infarct reduction and prevention of RI in a model of ischemia and reperfusion (18).

Recently published data suggest that adiponectin is a potential inhibitor of RI (19). Therefore, we conducted a study to assess the safety and efficiency of intracoronary downstream administration of adiponectin during reperfusion in terms of the reduction of the infarct size in the pig model of ischemia reperfusion injury.

\section{Materials and methods}

Closed chest acute ischemia and reperfusion. Eighteen Polish domestic pigs of both genders were enrolled. Animals were sedated with intramuscular ketamine hydrochloride (20 mg/kg) and xylazine ( $2 \mathrm{mg} / \mathrm{kg}$ ). Afterwards, animals were intubated and general anesthesia was performed with a bolus administration of intravenous propofol (20-40 mg) followed by a continuous infusion with $2-4 \mathrm{mg} / \mathrm{kg} / \mathrm{h}$. A vascular sheath (6F) was placed in the right femoral artery for arterial access. Subsequently, the animals were heparanized with a bolus amount of $100 \mathrm{IU} / \mathrm{kg}$. After initial coronary angiography and ventriculography, a 60-min total occlusion of the left anterior descending coronary artery (LAD) distal to the first diagonal branch was induced with an over the wire (OTW) balloon catheter. Acute ischemia was confirmed by ST elevations in the ECG. The animals were randomized just before reperfusion in a 1:1 ratio into the study group which were infused with intracoronary $5 \mathrm{ml}$ downstream adiponectin $(10 \mu \mathrm{g} / \mathrm{ml})$ through the central lumen of the OTW catheter 5 min prior to reperfusion and into the control group which were given $5 \mathrm{ml}$ saline $(0.9 \% \mathrm{NaCl})$ in the same way. After 60 min the balloon was deflated and the normal blood flow in the target artery was documented by angiography. The procedure was finished $30 \mathrm{~min}$ after reperfusion when control coronary angiography and ventriculography were performed. Animals were sacrificed after $48 \mathrm{~h}$ of reperfusion with a pentobarbital overdose. The study protocol was approved by the local ethics committee for animal research (no. 6/06, Katowice, Poland). The experiments were performed between 2007-2009 according to the Guide for the Care and Use of Laboratory Animals published by the US National Institutes of Health (NIH Publication No. 85-23, revised 1996).
Infarction and viable myocardium determination. Before euthanasia, a medial LAD was again occluded with a balloon catheter. Subsequently, $200 \mathrm{ml}$ of $1 \%$ Evans Blue dye was injected into the ascending aorta. The animals were then sacrificed and the hearts were explanted, frozen for $2 \mathrm{~h}$ and cut into $5 \mathrm{~mm}$ slices. The slices were then incubated for half an hour in $1 \%$ 2,3,5-triphenyltetrazoline (TTC) at $37^{\circ} \mathrm{C}$. The infarct area (IA) was marked with white color, the area at risk (AAR) with red and perfused muscle with dark blue. The slices were weighed and photographed. The IA, AAR and the area of the whole myocardium were measured with the Cell $\mathrm{A}^{\mathrm{TM}}\left(\operatorname{Sun}^{\circledR}\right)$ software.

Angiography and ventriculography. Angiography and ventriculography was performed before coronary artery occlusion, and then $30 \mathrm{~min}$ and $48 \mathrm{~h}$ after reperfusion to assess coronary flow, myocardial perfusion and left ventricle ejection fraction (LVEF\%). They were recorded in DICOM format (digital imaging and communications in medicine) in two contralateral projections: left anterior oblique $60^{\circ}$ and right anterior oblique $30^{\circ}$. The LVEF was assessed by a blinded operator with quantitative ventriculography (CMS, Medis Medical) from the following quotation: $\mathrm{LVEF}=\left(\mathrm{EDA}_{\mathrm{m}}-\mathrm{ESA}_{\mathrm{m}}\right) / \mathrm{EDA}_{\mathrm{m}}$ $100 \%$, in which $\mathrm{EDA}_{\mathrm{m}}$ was defined as the mean end diastolic area and $\mathrm{ESA}_{\mathrm{m}}$ as the mean end systolic area calculated from both projections. The blood flow in the infarct-related artery was assessed with the TIMI scale (thrombolysis in myocardial infarction) and TFC (TIMI frame count). Myocardial tissue perfusion was analyzed with the TMPG scale (TIMI myocardial perfusion grade). The angiogram was qualified as normal if both TIMI and TMPG were graded with 3 points.

Biochemical markers. The blood for biochemical evaluations was drawn before ischemia (0 h), and at 12, 24 and $48 \mathrm{~h}$ after reperfusion. At these time-points the concentration of troponin I, TNF- $\alpha$, interleukin 10 (IL-10) and high sensitive C-reactive protein (hs-CRP) were evaluated. For these assessments, a porcine-specific ELISA technique was utilized.

Determination of apoptosis. Apoptosis enhancement in the viable myocardium (AAR tissue) was determined by the TUNEL technique. For this, the In Situ Cell Death Detection $\mathrm{kit}^{\mathrm{TM}}$ (Roche) was utilized for recognition of the single and double DNA strand ruptures. The apoptosis enhancement was defined as the number of TUNEL positive signals found in 100 nuclei of ten randomly chosen specimen regions.

Histological analysis. Specimens from AAR and IA were collected. They were cut into $4 \mu \mathrm{m}$ slices and stained with hematoxylin and eosin. The severity of inflammation was expressed in a four grade quantitative scale (0-3). Zero was defined as no inflammation; 1 as focal; 2 as multifocal or diffuse; and 3 as severe.

Severe arrhythmia. Incidence of at least one episode of ventricular fibrillation (VF) or sustained ventricular tachycardia (VT) requiring defibrillation during the whole reperfusion period was defined as severe arrhythmia. Arrhythmias before the procedure and during the ischemic period were excluded from the analysis. 


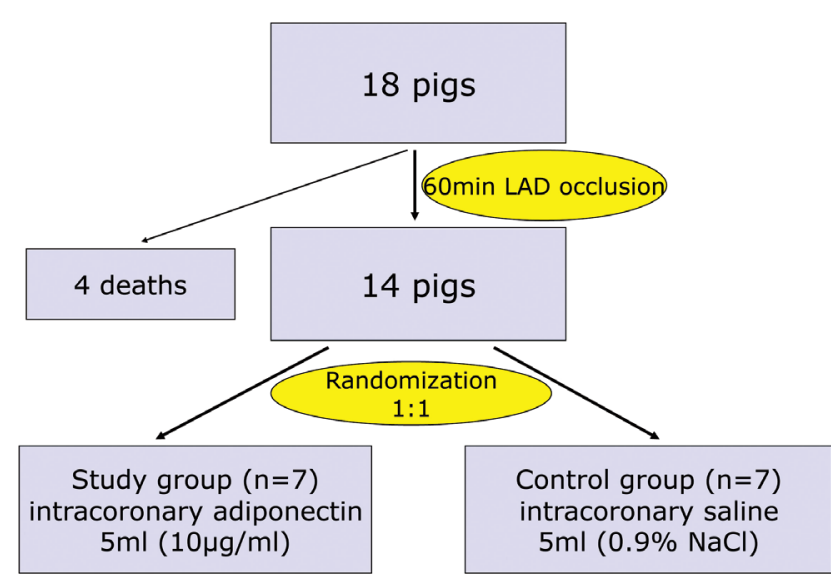

Figure 1. Study algorithm.

Study endpoints and definitions. The primary endpoint of this study was IA expressed as a percentage of AAR (IA/AAR\%). The IA was the secondary endpoint and was defined as the percentage of the area marked with TTC (necrotic area) over the area of the whole myocardium. AAR was defined as the percentage of the viable myocardium compared to the whole myocardium. The concentration or the activity of biochemical markers (troponin I, TNF- $\alpha$ and IL-1) angiographic parameters, the severity of apoptosis and inflammation were also considered as secondary endpoints.

Statistics. The parametric data were expressed as the mean value \pm standard deviation (SD). The non-parametric data were presented as a number and a percentage. Morphometric, angiographic and biochemical data were compared using the one-way analysis of variance (ANOVA). Variance uniformity was verified with the Levene test. In the cases of a skewed distribution or of a non-uniformity of variance, the nonparametric Kruskal-Wallis test was used. The inflammation score and apoptosis were analyzed by the Mann-Whitney U test. The incidence of severe arrhythmias and of coronary perfusion abnormalities were compared using the Fisher's test. p-values of $<0.05$ were considered statistically significant. All tests and graphs were performed using the MedCalc 9.1.0.1 (MedCalc Software). The hypothesis of this study was that adiponectin is superior in reducing infarct size in comparison to the placebo. The adiponectin superiority was considered to be significant, with an $\alpha$-value $<0.05$ and a power of 0.9 if $10 \pm 5 \%$ infarct salvage was achieved.

\section{Results}

A total of eighteen pigs were subjected to 60 min acute ischemia as described in the methods section. During this period 4 animals died because of fatal arrhythmia and were excluded from further analysis. Just before the reperfusion, the remaining animals were randomized either to the control group $(n=7)$ or to the study group (n=7) (Fig. 1). There were no differences between the study groups with regard to baseline characteristics such as gender (male $42.1 \%$ vs. $71.4 \%$; $\mathrm{p}=0.59)$, weight $(41.1 \pm 6.8 \mathrm{~kg}$ vs. $44.4 \pm 5.7 \mathrm{~kg} ; \mathrm{p}=0.34)$ and the pharmacological regimen before reperfusion.

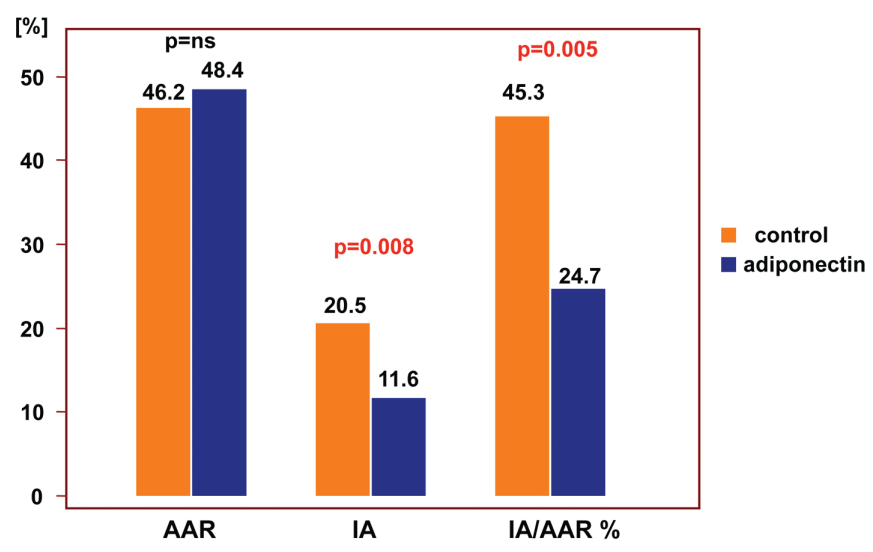

Figure 2. Infarct size morphometric analysis. AAR, area at risk; IA, infarct area.

Morphometric analysis. The AAR were comparable in both groups $(46.2 \pm 9.9 \%$ vs. $48.4 \pm 6.2 \%$; $=0.63)$. The primary endpoint of this study, IA/AAR\%, was significantly lower in the study group when compared to the control $(24.7 \pm 14.0 \%$ vs. $45.3 \pm 22.5 \% ; \mathrm{p}=0.005)$. Therefore, the hypothesis criteria for the superiority of adiponectin over the placebo were met. The secondary endpoint (IA) was also significantly lower in the adiponectin group $(11.6 \pm 5.0 \%$ vs. $20.5 \pm 5.6 \%$; $\mathrm{p}=0.008)$ (Fig. 2).

Biochemical evaluation of infarct size. The quantitative morphometric and hemodynamic findings were confirmed by the biochemical evaluations. Peak troponin I concentrations 12,24 and $48 \mathrm{~h}$ following reperfusion were significantly lower in the control group (Fig. 3A). The area under the curve was also significantly lower in the adiponectin group when compared to the control (3981.6 \pm 1542 vs. $6932.1 \pm 685$; MannWhitney U test: $\mathrm{p}=0.0012$ ).

Inflammatory markers. The concentrations of the inflammatory markers, TNF- $\alpha$, and IL-10 were not different between the study groups at any of the time-points examined (Fig. 3C and D, respectively). Only the hs-CRP value was significantly higher in the adiponectin group $48 \mathrm{~h}$ after reperfusion (3321 \pm 1272 vs. $1825 \pm 1015 \mathrm{ng} / \mathrm{ml} ; \mathrm{p}=0.03$ ) (Fig. 3B).

Quantitative ventriculography and coronary angiography. The LVEF was similar at baseline $(60.6 \pm 9.1 \%$ vs. $54.3 \pm 6.7 \%$; $\mathrm{p}=0.17)$ and $30 \mathrm{~min}$ after reperfusion $(49.1 \pm 7.5 \%$ vs. $44.1 \pm 6.4 \%$; $\mathrm{p}=0.2$ ). However, at $48 \mathrm{~h}$, the LVEF improved only in the adiponectin group and was significantly higher than in the control group $(51.4 \pm 8.5 \%$ vs. $33.9 \pm 8.6 \% ; \mathrm{p}=0.002)$ (Fig. 4). There was no difference in the epicardial TIMI flow, or in the microcirculatory flow expressed as TMPG. On the other hand, the epicardial flow in the TFC 25 scale was lower in the study group than in the control group $(19.7 \pm 3.9$ vs. $30.6 \pm 12.4 ; \mathrm{p}=0.025$ ).

Histopathological analysis. Profound myocyte structural abnormalities were found in the IA of both groups. Specifically, inflammatory infiltrations were observed both around cardiomyocytes and blood vessels, necrotic foci local- 
A

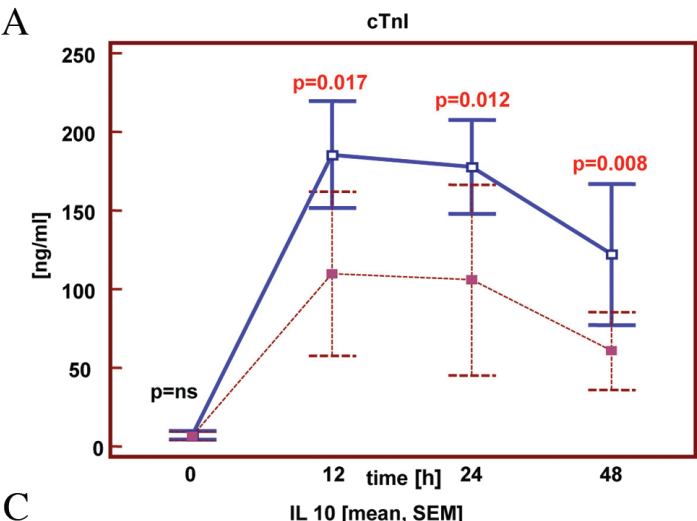

$\mathrm{C}$

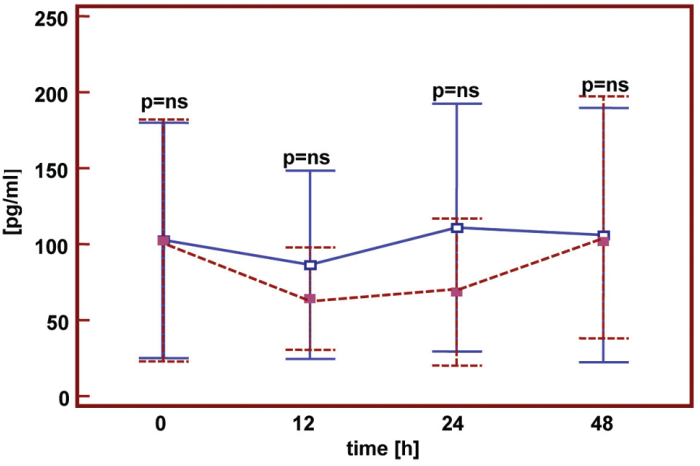

B

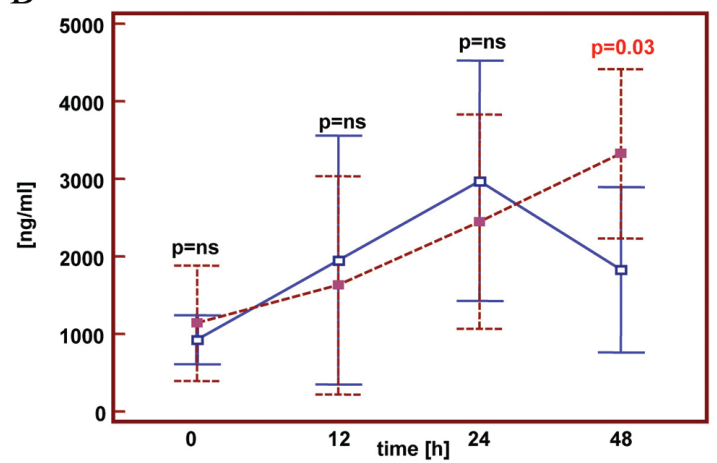

$\mathrm{D}$

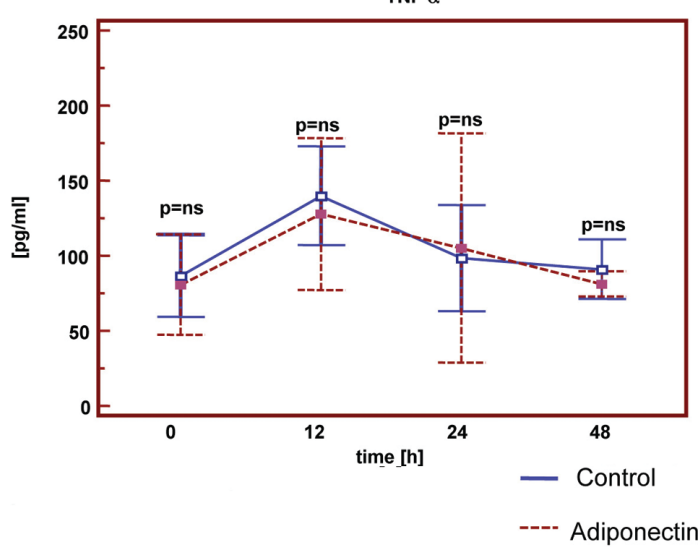

Figure 3. Infarction and inflammation markers. (A) troponin, (B) hs-CRP, (C) interleukin-10 (IL-10) and (D) TNF- $\alpha$.

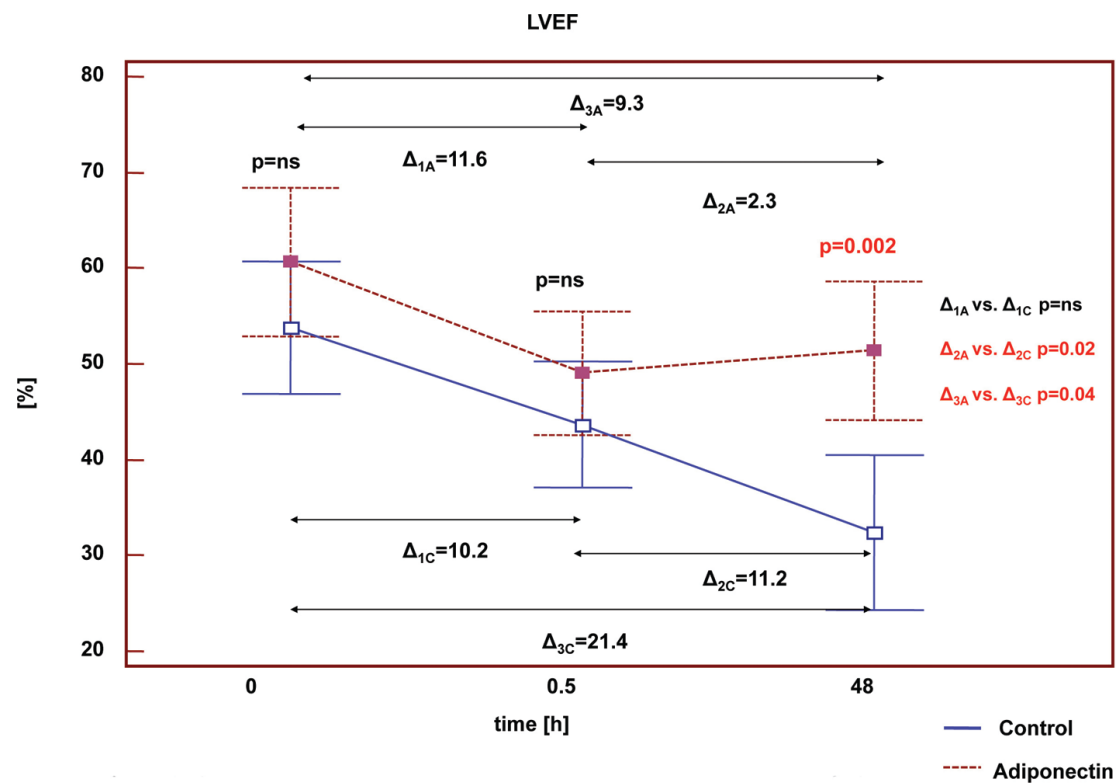

Figure 4. Left ventricular ejection fraction (LVEF\%): baseline, $0.5 \mathrm{~h}$ and $48 \mathrm{~h}$ after reperfusion in both groups. $\Delta \mathrm{XA}$, adiponectin; $\Delta \mathrm{XC}$, control.

ized mainly around vessels and only solitary normal tissue fragments. The analysis of inflammation in the AAR showed significantly less inflammation foci in the study group. The inflammation score in the IA was not different between the control and study groups $(1.83 \pm 1.1$ vs. $2.14 \pm 1.9 ; \mathrm{p}=0.53)$, however, it was significantly lower in the AAR of the study group ( $1.83 \pm 1.3$ vs. $0.14 \pm 0.38$; $\mathrm{p}=0.02$ ) (Fig. 5 ).

Apoptosis enhancement evaluation. Apoptosis enhancement in the viable myocardium of the AAR was evaluated by the 


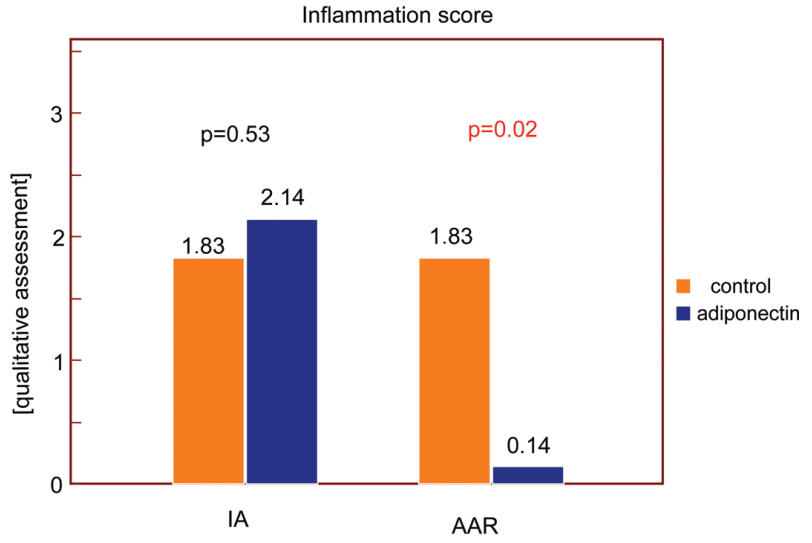

Figure 5. Severity of inflammation expressed in a quantitative scale. 0, no inflammation; 1 , focal; 2 , multifocal or diffuse; and 3, severe.

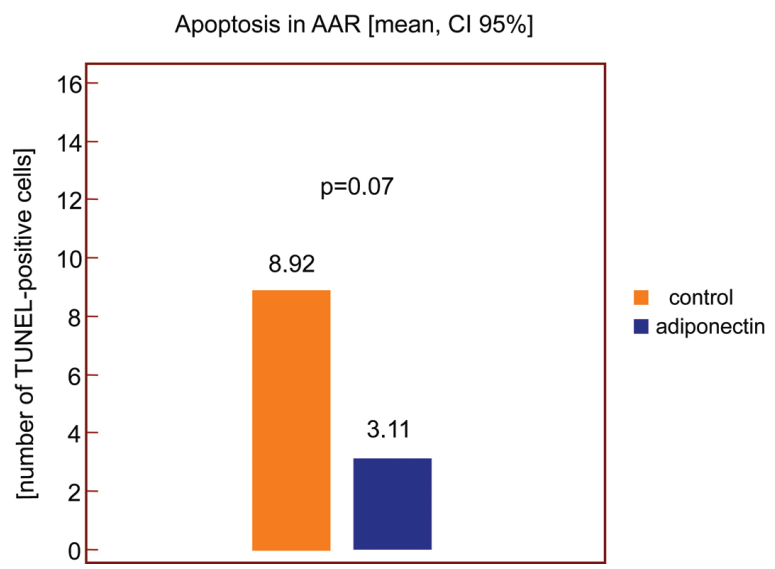

Figure 6. Enhancement of apoptosis in the area at risk expressed as the number of TUNEL-positive cells.

TUNEL method and resulted in a trend toward a lower number of TUNEL positive cells per 100 nuclei in the adiponectin group when compared to the control group, which was not statistically significant $(3.11 \pm 2.3$ vs. $8.92 \pm 6.3 ; \mathrm{p}=0.07)$ (Figs. 6 and 7).
Ventricular arrhythmias. Ventricular fibrillation requiring defibrillation after reperfusion occurred in one animal in the study group and in two animals in the control group (14.2\% vs. $28.5 \%$; $\mathrm{p}=0.1$.

\section{Discussion}

Our study is the first that demonstrates an almost 50\% reduction of the infarct size with adiponectin administration, especially at reperfusion, in the porcine model of ischemia reperfusion when compared with placebo. The myocardial salvage was confirmed by the lower troponin I concentration at all the timepoints examined in our study and, as a consequence, by a lower area under the curve. Moreover, adiponectin administration prevented further decrease of the LVEF after $48 \mathrm{~h}$. Adiponectin also improved epicardial blood flow according to the objective TFC 25 scale, although there was no difference in myocardial microcirculatory perfusion expressed as the TMPG scale. These clinical and laboratory results are supported by the evaluation of apoptosis in the viable myocardium, which revealed a trend toward a lower number of TUNEL-positive cells. Despite the fact that serum adiponectin concentration negatively correlates with serum inflammatory markers $(20,21)$ in the present study there was no difference in IL-10 and TNF- $\alpha$ concentrations between the two groups. On the other hand, the CRP level at $48 \mathrm{~h}$ after reperfusion was higher in the study group. These results may suggest that intracoronary downstream delivery of adiponectin does not influence the systemic inflammatory response but only the local response, and therefore, it is not distinguishable in the peripheral blood. This may also be related to high myocardial clearance and deficit of adiponectin in the myocardium during infarction. This hypothesis seems to be confirmed by a pathological examination in which a lower inflammatory response was observed in the viable myocardium (AAR) of the study group; however, there was no difference in the severity of inflammation in the IA. Similarly, the apoptosis enhancement was decreased in the area around the infarct. These findings indicate that adiponectin with its anti-inflammatory and anti-apoptotic properties reduces the infarct size in the viable myocardium area as a consequence of RI inhibition.

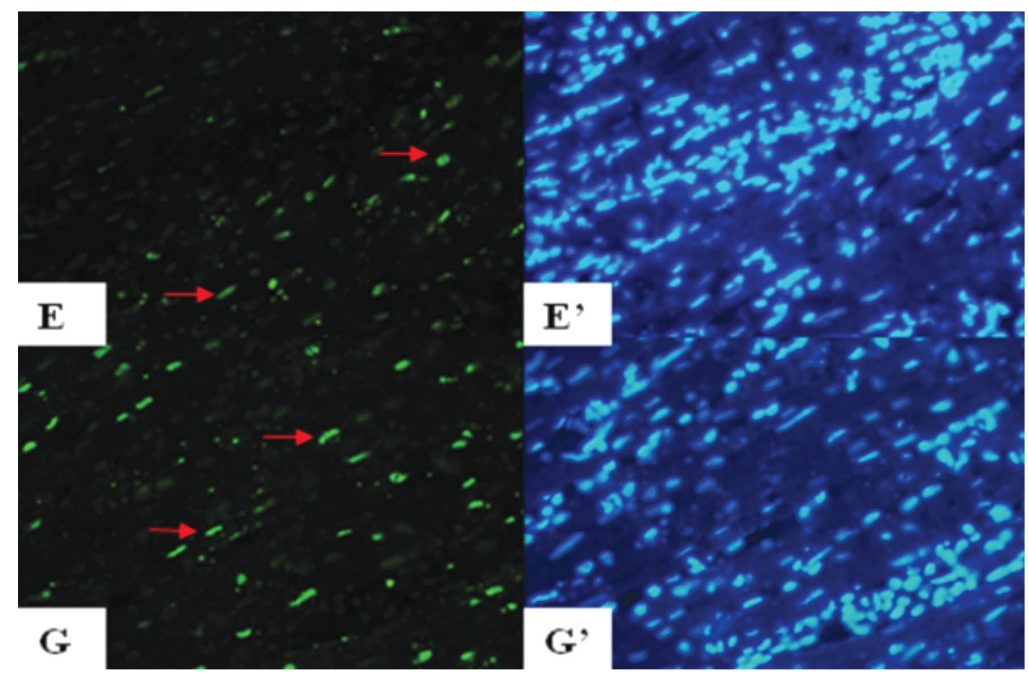

Figure 7. Apoptosis enhacement. E, TUNEL and E', DAPI with adiponectin; G, TUNEL and G', DAPI without adiponectin. 
The porcine model chosen in our study seems to be optimal in translational research of myocardial ischemia and reperfusion. The minimally invasive, 'closed chest', 60 min occlusion of the medial LAD generates a large infarct size, with a low mortality rate due to severe arrhythmias (22). Furthermore, it is crucial that the substance is delivered just before reperfusion, since the cascade of RI begins together with blood flow restoration to the infarct-related artery $(23,24)$. The use of clinically approved OTW catheters for local drug delivery into the ischemic area is safe and feasible. Therefore, it can be easily introduced into the clinical practice.

The outcome of this study is consistent with and extends the current knowledge about the role of adiponectin as a cardiovascular risk factor. A low level of adiponectin is associated with hypertension (25), obesity $(26,27)$, diabetes (28) and most importantly for this study, with occurrence of an acute coronary syndrome. The hypothesis generated by Shibata et al (18) in the rat myocardial reperfusion infarction model was confirmed in our study. Moreover, the results presented in this report are consistent with those recently published by Kondo et al (19) with a similar study design. Both studies were initiated at about the same time by different investigators in different facilities. However, in our study we used porcine adiponectin, administered immediately before reperfusion, which more closely mimics the potential clinical application, compared to the administration of adiponectin by continuous infusion and from the beginning of ischemia. With this method of local drug delivery and reduced inflammation only in the AAR, we demonstrate that its effectiveness is specifically related to amelioration of RI. In clinical conditions after successful PCI, targeting this pathological process is possible and therefore preventable. In the previously cited study, it was undetermined whether adiponectin reduces infarct size by modulating acute ischemia or by modulating RI. Therefore, any further clinical implications and applications remain unclear. Moreover, we have expanded on their findings by demostrating improvement in the ejection fraction two days after reperfusion, as well as an improvement in myocardial perfusion.

Due to the fact that positive infarct reduction animal studies do not often translate into clinical trials (29) the consensus of the National Heart, Lung and Blood Institute (NHLBI) states that only agents that have been conclusively shown to be cardioprotective in animal experiments by multiple investigators should be investigated in the clinical setting (30). Our study together with the previously described study by Kondo et al (19) meet this NHLBI criterion by showing very promising results with adiponectin in the porcine myocardial infarct reduction.

In conclusion, the local delivery of the adipose-derived hormone, adiponectin, to the infarcted porcine myocardium is safe and successful in reducing the infarct size caused by RI. Additionally, the overall availability and simplicity of the presented method, makes the clinical introduction of adiponectin into the first in-human trial reasonable and promising.

\section{Acknowledgements}

This study was supported by grant N40210431/3212 of the Ministry of Science and Higher Education.

\section{References}

1. Rogers WJ, Canto JG, Lambrew CT, et al: Temporal trends in the treatment of over 1.5 million patients with myocardial infarction in the US from 1990 through 1999: the National Registry of Myocardial Infarction 1,2 and 3. J Am Coll Cardiol 36: 2056-2063, 2000.

2. Zahn R, Schiele R, Schneider S, et al: Decreasing hospital mortality between 1994 and 1998 in patients with acute myocardial infarction treated with primary angioplasty but not in patients treated with intravenous thrombolysis. Results from the pooled data of the Maximal Individual Therapy in Acute Myocardial Infarction (MITRA) Registry and the Myocardial Infarction Registry (MIR). J Am Coll Cardiol 36: 2064-2071, 2000.

3. Mehran R, Brodie B, Cox DA, et al: The Harmonizing Outcomes with RevasculariZatiON and Stents in Acute Myocardial Infarction (HORIZONS-AMI) Trial: study design and rationale. Am Heart J 156: 44-56, 2008.

4. Spencer FA, Meyer TE, Gore JM and Goldberg RJ: Heterogeneity in the management and outcomes of patients with acute myocardial infarction complicated by heart failure: the National Registry of Myocardial Infarction. Circulation 105: 2605-2610, 2002.

5. Vaur L, Danchin N, Genes N, et al: Epidemiology of myocardial infarction in France: therapeutic and prognostic implications of heart failure during the acute phase. Am Heart J 137: 49-58, 1999.

6. Levy D, Kenchaiah S, Larson MG, et al: Long-term trends in the incidence of and survival with heart failure. N Engl J Med 347: 1397-1402, 2002.

7. Park JL and Lucchesi BR: Mechanisms of myocardial reperfusion injury. Ann Thorac Surg 68: 1905-1912, 1999.

8. Yellon DM and Hausenloy DJ: Myocardial reperfusion injury: $\mathrm{N}$ Engl J Med 357: 1121-1135, 2007.

9. Braunwald E and Kloner RA: Myocardial reperfusion: a doubleedged sword? J Clin Invest 76: 1713-1719, 1985.

10. Piper HM, Meuter K and Schafer C: Cellular mechanisms of ischemia-reperfusion injury. Ann Thorac Surg 75: 644-648, 2003.

11. Ross AM, Gibbons RJ, Stone GW, Kloner RA and Alexander RW: A randomized, double-blinded, placebo-controlled multicenter trial of adenosine as an adjunct to reperfusion in the treatment of acute myocardial infarction (AMISTAD-II). J Am Coll Cardiol 45: 1775-1780, 2005.

12. Bates E, Bode C, Costa M, et al: Intracoronary KAI-9803 as an adjunct to primary percutaneous coronary intervention for acute ST-segment elevation myocardial infarction. Circulation 117 : 886-896, 2008

13. Cheng KK, Lam KS, Wang Y, et al: Adiponectin-induced endothelial nitric oxide synthase activation and nitric oxide production are mediated by APPL1 in endothelial cells. Diabetes 56: 1387-1394, 2007.

14. Motoshima H, Wu X, Mahadev K and Goldstein BJ: Adiponectin suppresses proliferation and superoxide generation and enhances eNOS activity in endothelial cells treated with oxidized LDL. Biochem Biophys Res Commun 315: 264-271, 2004.

15. Nakanishi S, Yamane K, Kamei N, Nojima H, Okubo M and Kohno N: A protective effect of adiponectin against oxidative stress in Japanese Americans: the association between adiponectin or leptin and urinary isoprostane. Metabolism 54: 194-199, 2005.

16. Ouedraogo R, Gong Y, Berzins B, et al: Adiponectin deficiency increases leukocyte-endothelium interactions via upregulation of endothelial cell adhesion molecules in vivo. J Clin Invest 117: 1718-1726, 2007.

17. Kobayashi H, Ouchi N, Kihara S, et al: Selective suppression of endothelial cell apoptosis by the high molecular weight form of adiponectin. Circ Res 94: 27-31, 2004.

18. Shibata R, Sato K, Pimentel DR, et al: Adiponectin protects against myocardial ischemia-reperfusion injury through AMPKand COX-2-dependent mechanisms. Nat Med 11: 1096-1103, 2005.

19. Kondo K, Shibata R, Unno K, et al: Impact of a single intracoronary administration of adiponectin on myocardial ischemia/ reperfusion injury in a pig model. Circ Cardiovasc Interv 3: 166-174, 2010.

20. Ouchi N, Kihara S, Funahashi T, Matsuzawa Y and Walsh K: Obesity, adiponectin and vascular inflammatory disease. Curr Opin Lipidol 14: 561-566, 2003. 
21. Ouchi N, Kihara S, Funahashi T, et al: Reciprocal association of C-reactive protein with adiponectin in blood stream and adipose tissue. Circulation 107: 671-674, 2003.

22. Suzuki Y, Lyons JK, Yeung AC and Ikeno F: In vivo porcine model of reperfused myocardial infarction: in situ double staining to measure precise infarct area/area at risk. Catheter Cardiovasc Interv 71: 100-107, 2008.

23. Zweier JL, Flaherty JT, Weisfeldt ML: Direct measurement of free radical generation following reperfusion of ischemic myocardium. Proc Natl Acad Sci USA 84: 1404-1407, 1987.

24. Arroyo CM, Kramer JH, Dickens BF and Weglicki WB: Identification of free radicals in myocardial ischemia/reperfusion by spin trapping with nitrone DMPO. FEBS Lett 221: 101-104, 1987.

25. Iwashima Y, Katsuya T, Ishikawa K, et al: Hypoadiponectinemia is an independent risk factor for hypertension. Hypertension 43: 1318-1323, 2004.
26. Arita Y, Kihara S, Ouchi N, et al: Paradoxical decrease of an adipose-specific protein, adiponectin, in obesity. Biochem Biophys Res Commun 257: 79-83, 1999.

27. Furukawa S, Fujita T, Shimabukuro M, et al: Increased oxidative stress in obesity and its impact on metabolic syndrome. J Clin Invest 114: 1752-1761, 2004.

28. Hotta K, Funahashi T, Arita Y, et al: Plasma concentrations of a novel, adipose-specific protein, adiponectin, in type 2 diabetic patients. Arterioscler Thromb Vasc Biol 20: 1595-1599, 2000.

29. Bolli R BL, Gross G, Mentzer R Jr, Balshaw D and Lathrop DA: Myocardial protection at a crossroads: the need for translation into clinical therapy. Circ Res 95: 125-134, 2004.

30. Baxter GF, Hale SL, Miki T, et al: Adenosine A1 agonist at reperfusion trial (AART): results of a three-center, blinded, randomized, controlled experimental infarct study. Cardiovasc Drugs Ther 14: 607-614, 2000 